\title{
Normal forms of linear second order partial differential equations on the plane
}

\author{
Alexey Davydov ${ }^{1,2,3}$ \\ ${ }^{1}$ Department of Mathematics, The National University of Science and Technology MISiS, Moscow 119049, Russia; \\ 2 Department of Theory of Dynamical Systems, Lomonosov Moscow State University, Moscow 119991, Russia; \\ ${ }^{3}$ International Institute for Applied Systems Analysis, Laxenburg A-2361, Austria \\ Email:davydov@mi.ras.ru
}

Received October 3, 2017; accepted April 18, 2018; published online September 10, 2018

\begin{abstract}
The paper is devoted to the theory of normal forms of main symbols for linear second order partial differential equations on the plane. We discuss the results obtained in the last decades and some problems, which are important both for the development of this theory and the applications. The reduction theorem, which was used to obtain many of recent results in the theory, is included in the paper in the parametric form together with proof. There is a feeling that the theorem still has potential to get progress in the solution of open problems in the theory.
\end{abstract}

Keywords normal form, mixed type partial differential equation, main symbol

MSC(2010) 34A09, 34C20

Citation: Davydov A. Normal forms of linear second order partial differential equations on the plane. Sci China Math, 2018, 61: 1947-1962, https://doi.org/10.1007/s11425-017-9303-0

\section{Introduction}

The beginning of the theory of normal forms of linear second order partial differential equations on the plane goes back to the middle of the 18th century. At that time d'Alembert and Euler proposed the wave equation and the Laplace equation to describe the motion of the string and the velocity potential of an incompressible fluid, respectively. After the appearance these forms, which represent the elliptic and hyperbolic type equations, are actively used in the analysis of various applied problems, and now the ones are well-known and usually are included in standard university courses of partial differential equations. The main symbol of the equation

$$
a(x, y) u_{x x}+b(x, y) u_{x y}+c(x, y) u_{y y}=0
$$

with smooth coefficients $a, b, c$ could be reduced to these forms locally near any point of its hyperbolicity and ellipticity, respectively, i.e., where the discriminant $D, D=b^{2}-4 a c$, of this equation is positive and negative, respectively, by smooth change of coordinates and multiplication on an appropriate smooth nonvanishing function. For a generic triplet $(a, b, c)$ in smooth or sufficiently smooth fine Whitney topology the zero level discriminant is either empty or is a smoothly embedded curve in the plane. Thus for a 
generic equation the wave and Laplace equations, i.e., $u_{x x}-u_{y y}=0$ and $u_{x x}+u_{y y}=0$, present normal forms of the main symbol of (1.1) near a point outside this line.

This line is called type change line because near any its point the equation has points of its both ellipticity and hyperbolicity. Equation (1.1), which changes type in the domain, is named of mixed type.

The systematic studies of mixed type partial differential equations go back to the treatise (see [32]), in which Tricomi provided the ideology and motivation for many studies in this area in the 20th century. In this treatise, Tricomi [32] considered an equation near a point $P$ of the type change line, which is non-degenerate zero of the descriminant, i.e., $D(P)=0$ and $d D(P) \neq 0$, and at which the equation characteristic direction $d y: d x$ defined by the equation

$$
a(x, y) d y^{2}-b(x, y) d x d y+c(x, y) d x^{2}=0
$$

is not tangent to the line. Near such a point he proposed for (1.1) a normal form of the main symbol

$$
u_{y y}+y u_{x x}=0
$$

up to smooth change of coordinates and multiplication on a non-vanishing smooth function. In the form this equation changes type on the $x$-axis, and it is of elliptic and hyperbolic type in domains $y>0$ and $y<0$, respectively. Also, it was shown that the equation in the normal form has two outgoing characteristics at each point $x_{0}$ of the abscissa axis. The characteristics lie in the domain $y \leqslant 0$ and have the form $9\left(x-x_{0}\right)^{2}=-4 y^{3}$.

For (1.3), Tricomi [32] formulated the new type of the boundary value problem in the domain bounded by intersecting characteristics outgoing from two points of the type change line and by a smooth arc, which lies in the domain $y \geqslant 0$ and connects these points. For boundary conditions of Dirichlet type defined on this arc and on one of the two arcs of characteristics he proved the theorem of existence and uniqueness of the solution. Now this problem is named as Tricomi one (see also $[27,28]$ ).

In the treatise, Tricomi [32] also provided the ground for the normal form (1.3), but his proof has a gap. The correct proof for this form was done by Cibrario [7]. As we noted above the Tricomi's results were actively used during the last century, especially in applications connected with the analysis of the transonic gas flow.

The next step in the theory of normal forms of generic mixed type partial differential equations on the plane was done essentially later, namely, in the last quarter of the previous century and at the beginning of this one. This paper is devoted to these recent results and also the reduction theorem, different variants of which were used to obtain these results.

\section{The equation surface, folded singular points and reduction theorem}

Firstly, we present an additional motivation to study binary equations on the plane and their normal forms and also the main tool, namely, the reduction theorem, which was used to obtain recent results in the area.

\subsection{Lifted direction field}

The characteristics of mixed type equations are defined by (1.2), which is a binary differential equation. There are many other interesting practical situations when the integral curves of such equations play the important role. Next, two examples are illustrated as follows.

Example 2.1. Consider on the plane the simplest dynamic inequality

$$
(\dot{x}-v(x, y))^{2}+(\dot{y}-u(x, y))^{2} \leqslant f(x, y),
$$

which describes in the domain $f \geqslant 0$ with flow $(v(x, y), u(x, y))$ a motion of the non inertial object with proper possibilities for the motion with velocity $\sqrt{f}$ in any direction. At a point, where the flow is too strong, namely, when

$$
v^{2}+u^{2} \geqslant f(x, y) \geqslant 0
$$




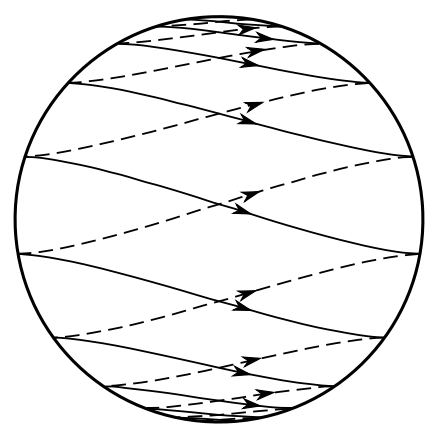

Figure 1 The net of integral curves of (2.2)

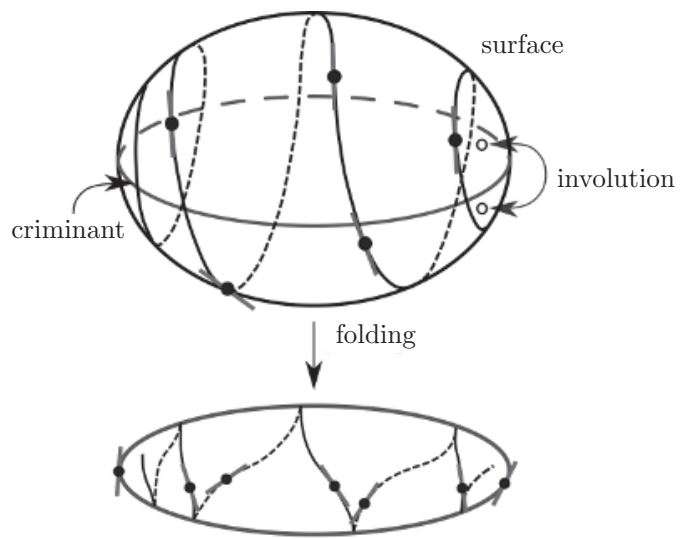

Figure 2 The equation surface, direction field, folding, involution and criminant

the directions of admissible velocities of the object form a cone, the boundary of which is the union of two directions. These directions are called the limiting ones at the point. The field of limiting directions is also defined by the respective binary differential equation, which could be easily calculated from (2.1).

The domain of the dynamic inequality

$$
(\dot{x}-2)^{2}+(\dot{y})^{2} \leqslant 1-x^{2}-y^{2}
$$

is the disk $1-x^{2}-y^{2} \geqslant 0$, and its net of integral curves looks like Figure 1. The small arrows on these curves indicate the direction of the motion along the curves with admissible velocities. In this case local normal forms of binary differential equations play an important role in the analysis of controllability and the singularities of controllability of the respective inequalities (see $[10,19]$ ).

Example 2.2. In the three-dimensional Euclidian space the asymptotic lines net on the graph $z=$ $f(x, y)$ of a smooth function $f$ is described in coordinates $x$ and $y$ on this graph by the equation

$$
f_{x x}(x, y) d x^{2}+2 f_{x y}(x, y) d x d y+f_{y y}(x, y) d y^{2}=0 .
$$

That is also the binary differential equation. The net of integral curves of this equation could also look like Figure 1, in particular in the situation, when the hyperbolicity domain on this graph has just appeared (see [4]).

Remark 2.3. $\quad$ Note that Figure 1 also presents a characteristic net of the linear mixed type equation on the plane

$$
u_{x x}+K(x, y)\left(1-x^{2}-y^{2}\right) u_{y y}=0
$$

with some smooth negative function $K$, which could be easily found inside the disk by calculating the limiting direction field for (2.2). 
One of the approaches to study the integral curves of binary differential equations was proposed by Poincare in the context of analysis of behaviour of the ones for the case of general differential equations, which is not resolved with respect to the derivative. The main idea was to lift the multi valued direction field of such equation to the single valued direction field on the equation surface, which is defined by the equation in the space of directions on the plane in Figure 2. A binary differential equation defines in this space the equation surface, which is smooth for typical equations and under the projection along the direction axis could have only singular points of type Whitney fold. These singular points form criminant of the equation.

The equation direction field is defined on this surface by standard contact structure in the direction space as the intersection of the contact plane and the tangent plane to the surface. At the points, where these two planes coincide, the field has singular points. On Figure 2 one can see two such points of type focus. The restriction of the projection along the direction axis $(x, y, d y: d x) \mapsto(x, y)$ to the equation surface is called equation folding. The equation folding defines on the surface the folding involution that permutates two points on the surface having the same image under this folding.

The image of the criminant under the equation folding is called discriminant curve, and it is exactly the type change line for partial differential equations and corresponds to the parabolic line of the asymptotic lines net.

Example 2.4. The characteristic direction field of Cibrario-Tricomi equation in the form $d y^{2}-x d x^{2}$ $=0$ is defined in the half-plane $x \geqslant 0$, and it is two-valued inside it. The lifted direction field on the equation surface $p^{2}=x, p=d y / d x$, is defined as $d y: d p=2 p^{2}: 1$ in coordinates $y$ and $p$ on it. The criminant and the discriminant curves are $p=0$ and $x=0$, respectively.

Example 2.5. $\quad$ For the equation $d y^{2}=\left(y+x^{2}\right) d x^{2}$ the lifted direction field on the equation surface with coordinates $x$ and $p$ is defined as $d x: d p=2 p:(p+2 x)$. The origin is the singular point of the field and also the critical point of the equation folding. The criminant and the discriminant curves are $p=0$ and $y=-x^{2}$, respectively.

The construction with lifting of the direction field gives a possibility to reduce the classification of characteristic net singularities of generic linear second order mixed type partial differential equations on the plane to such one for pairs of the equation folding involution and the equation direction field. This reduction gives a possibility to complete this classification by the end of the 20 th century in $[17,18]$. The matter is that for a typical binary differential equation outside the discriminant curve we have situations corresponding to points of ellipticity or hyperbolicity of partial linear second order equations on the plane, and so the classical Laplace or wave equation normal forms work here. In addition, near a point of the discriminant curve we arrive at a germ of a pair (the equation folding involution, the equation direction field) at the respective point. The Cibrario-Tricomi equation provides the normal form near a typical point of the discriminant curve, when the lifted direction field is not singular. In the next subsection we describe the reduction theorem, which gives a possibility to apply the known local normal forms for vector or direction fields (see [2]) to obtain the normal forms of such a pair for the case of binary differential equations near a singular point of the lifted direction field.

\subsection{Reduction theorem}

The reduction theorem we formulate here is in the parametric case. Instead for a single binary differential equation we consider a smooth family of such equations parameterized by the finite dimensional parameter $\epsilon$. All our discussions are local near the considered point of the discriminant curve, where the differential of the discriminant is not zero for a given value (= zero) of the parameter and the equation direction field is tangent to the curve. In general here we follow $[12,13]$.

Proposition 2.6 (See [13]). A smooth family of the equations

$$
a(x, y, \varepsilon) d y^{2}-2 b(x, y, \varepsilon) d x d y+c(x, y, \varepsilon) d x^{2}=0
$$

with a finite dimensional parameter $\epsilon$ near a point $P$ of the discriminant curve, $D(P)=0, d D(P) \neq 0$, 
where the direction field is tangent to the curve, takes the form

$$
d y^{2}+c(x, y, \epsilon) d x^{2}=0
$$

near the origin, where $c, c(O)=0=c_{x}(O) \neq c_{y}(O)$, is some new smooth function, after an appropriate selection near this point of smooth local coordinates foliated over the parameter and multiplication by some smooth non-vanishing function.

Proof. Firstly, we select near the point $P$ smooth local coordinates with the origin at this point such that the abscissa axis direction at the point is exactly the value of the equation direction field. That gives a possibility to rewrite $(2.5)$ in the form

$$
a(x, y, \varepsilon) p^{2}-2 b(x, y, \varepsilon) p+c(x, y, \varepsilon)=0,
$$

where $p=d y / d x$ and $a, b, c$ are smooth functions; $a(O) \neq 0=b(O)=c(O), c_{y}(O) \neq 0$ due to coordinate selection and the conditions $D(O)=0,\left|D_{x}(O)\right|+\left|D_{y}(O)\right| \neq 0$. Here, $O=(0,0,0)$. For a new coordinate $\tilde{y}, y=Y(x, \tilde{y}, \epsilon)$ and we have

$$
\frac{d y}{d x}=Y_{x}(x, \tilde{y}, \epsilon)+Y_{\tilde{y}}(x, \tilde{y}, \epsilon) \frac{d \tilde{y}}{d x} .
$$

Substituting that to our equation we get after simple transformations the equation

$$
Y_{\tilde{y}}^{2}\left(\frac{d \tilde{y}}{d x}\right)^{2}+2 Y_{\tilde{y}}\left[Y_{x}-b(x, Y, \epsilon)\right] \frac{d \tilde{y}}{d x}+c(x, Y, \epsilon)+Y_{x}^{2}-2 b(x, Y, \epsilon) Y_{x}=0,
$$

in which the second term is zero if the expression in square brackets is identically zero, i.e., $Y$ is a solution of the equation

$$
Y_{x}=b(x, Y, \epsilon) .
$$

For any given smooth initial conditions on the plane $x=0$ the last equation has the unique and smooth solution because the surface $x=0$ has no characteristic points of the equation. Selecting $Y(0, \tilde{y}, \epsilon)=\tilde{y}$ we could write the respective solution in the form

$$
Y(x, \tilde{y}, \epsilon)=\tilde{y}+x B(x, \tilde{y}, \epsilon),
$$

where $B$ is some smooth function, due to Hadamard lemma (see [3]). Substituting this solution to (2.7) and multiplying the result by $Y_{\tilde{y}}^{-2}$ we get the needed equation form (2.6) with some new function $c, c(O)=0=c_{x}(O) \neq c_{y}(O)$.

For (2.6) its folding involution $\sigma$ has the form

$$
(x, p) \mapsto(x,-p)
$$

in coordinates $x$ and $p=d y / d x$ on the equation surface, and the equation direction field could be defined on the equation surface by the vector field

$$
v:=\left(-2 p, c_{x}+p c_{y}\right)
$$

as it is easy to see. The origin is the singular point of this vector field, and, in addition, on the line $p=0$ of fixed points of the folding involution this field is either zero or has the direction of $p$-axis. In addition at a point $(x, p)$ of the equation surface the image of this field under the folding involution is

$$
\sigma_{*} v(x, p)=\left(2 p,-c_{x}+p c_{y}\right) .
$$

Hence the determinant of the matrix with columns $v$ and $\sigma_{*} v$ has at a point $(x, p)$ the value $4 p^{2} c_{y}$. Due to $c_{y}(O) \neq 0$ this determinant has exactly the second order zero on the line of fixed points of the folding involution. In particular the fields $v$ and $\sigma_{*} v$ are collinear only on the line. Based on that we introduce the following notion of compatibility. 
In the plane a vector field and a differentiable involution with a line of fixed points are called compatible at a point of the line if near this point the determinant of the matrix defined by the field and its image under the involution has the second order zero on the line. In the plane a direction field and a differentiable involution with a line of fixed points are called compatible at a point of the line if the field could be defined by a vector field being compatible with the involution at this point. Compatibility of germs is defined analogously.

Example 2.7. In the plane vector field $(x, \alpha y)$ with $\alpha>1$ and involution $(x, y) \mapsto\left(\frac{(\alpha+1) x-2 \alpha y}{\alpha-1}\right.$, $\left.\frac{2 x-(\alpha+1) y}{\alpha-1}\right)$ are compatible.

Two objects (functions or germs of functions, maps, etc.) are called $C^{r}$-equivalent along a differentiable vector field $v\left(=C_{v}^{r}\right.$-equivalent $)$ if they could be transformed into each other by $C^{r}$-diffeomorphism mapping integral curves of the field into themselves. For a finite parameter family of objects a $C_{v}^{r}$ equivalence is $C^{r}$-diffeomorphism preserving the natural fibration over the family's parameter $\epsilon$ and mapping the integral curves of the field $(v, \dot{\epsilon}=0)$ into themselves; $C_{v}^{r}$-equivalence is strong if, in addition, it preserves the parameter.

Theorem 2.8 (See [13]). Two germs at the origin of smooth families $\left(v, \sigma_{1}\right)$ and $\left(v, \sigma_{2}\right)$ of compatible pairs of vector fields and involutions with the same finite dimensional parameter and the surface of fixed points of involutions, which passes through the origin, are strongly $C_{v}^{\infty}$-equivalent, if for any fixed parameter value being sufficiently close to zero the field $v$ is transversal to the line of fixed points of involutions almost everywhere.

We call this statement the reduction theorem.

This theorem reduces the problem of local normal forms of binary differential equations for many generic cases to the theory of local normal forms of compatible pairs of the vector field and involution.

Main steps of the proof of this theorem are the same as in [9]. We include this proof here because the theorem is the main tool for deduction of normal forms for binary differential equations from the ones for vector fields.

To prove the theorem let us take near the origin smooth local coordinates $x, y$ and $\epsilon$ foliated over the parameter such that the family $\sigma_{1}$ and the surface of the fixed points of involutions take forms $(x, y, \varepsilon) \mapsto(x,-y, \varepsilon)$, and $y=0$, respectively. For such selection it is sufficient to take any smooth function $f, f(O)=0$, whose differential at the origin has non-zero values on the eigenvectors of the derivative $\sigma_{1, *}(O)$, and select new local coordinates $x=F+\sigma_{1}^{*} F, y=F-\sigma_{1}^{*} F$, and the same coordinate $\epsilon$.

According to the compatibility of $v=\left(v_{1}, v_{2}\right)$ and $\sigma_{1}$ we find that the function

$$
\left|\begin{array}{cc}
v_{1}(x, y, \varepsilon) & v_{2}(x, y, \varepsilon) \\
v_{1}(x,-y, \varepsilon) & -v_{2}(x,-y, \varepsilon)
\end{array}\right|=-v_{1}(x, y, \varepsilon) v_{2}(x,-y, \varepsilon)-v_{1}(x,-y, \varepsilon) v_{2}(x, y, \varepsilon)
$$

has exactly the second order zero on the surface $y=0$ of fixed points of involutions.

In particular on this surface $v_{1} v_{2} \equiv 0$. But the field $(v, \dot{\epsilon}=0)$ is transversal to the surface almost everywhere due to the theorem assumption, and so the last equality implies $v_{1}(x, 0, \varepsilon) \equiv 0$. In addition, $(0,1,0)$ is the eigendirection of the derivatives $\sigma_{1 *}, \sigma_{2 *}$ with the eigenvalue -1 at any point of the surface.

Hence these derivatives are the same at any point of the surface, and so in selected coordinates near the origin the family $\sigma_{2}$ could be written in the form

$$
(x, y, \varepsilon) \mapsto\left(x+y^{2} r(x, y, \varepsilon),-y+y^{2} s(x, y, \varepsilon), \varepsilon\right)
$$

with some smooth functions $r$ and $s$. Consequently there exist coordinates

$$
\zeta=x+y^{2} R(x, y, \varepsilon), \quad \eta=y+y^{2} S(x, y, \varepsilon)
$$

with some smooth functions $R$ and $S$ and the same $\epsilon$, in which the family $\sigma_{2}$ of involutions has a form

$$
(\zeta, \eta, \varepsilon) \mapsto(\zeta,-\eta, \varepsilon) .
$$


The rest part of the proof is based on the homotopical method proposed by Thom (see [3]). Locally near the origin consider the smooth deformation of coordinates

$$
\gamma_{t}:\left(\zeta_{t}, \eta_{t}, \varepsilon\right) \mapsto\left(\zeta_{t},-\eta_{t}, \varepsilon\right),
$$

where

$$
\zeta_{t}=x+t y^{2} R(x, y, \varepsilon), \quad \eta_{t}=y+t y^{2} S(x, y, \varepsilon)
$$

and the respective deformation of involution families $\sigma_{1}$ and $\sigma_{2}$ :

$$
\left(\zeta_{t}, \eta_{t}, \varepsilon\right) \mapsto\left(\zeta_{t},-\eta_{t}, \varepsilon\right)
$$

Denote by $V_{t}$ the respective infinitesimal deformation field, which is the velocity of motion of the point image under a smooth deformation of involution. It is clear that the considered deformation $V_{t}$ has zero component along the parameter axis.

Lemma 2.9. A field $V$ is the infinitesimal deformation field of a smooth deformation of family $\sigma$, which is fibered over the parameter, if and only if $\sigma_{*} V=-V$.

Lemma 2.10. For a deformation $g$ of the identity, which is fibered over the parameter, with a velocity $h$ the family of involutions $\sigma:(x, y, \varepsilon) \mapsto(x,-y, \varepsilon)$ is deformed with the velocity $h-\sigma_{*} h$.

These lemmas are analogous to the respective statements from $[9,11]$. They can be proved by a direct calculation. Due to that we omit the proofs of these lemmas here.

Due to Lemma 2.10 to prove the theorem it is sufficient locally near the interval $[0,1]$ of the $t$-axis to present the deformation velocity in the form

$$
V_{t}=f_{t} v-\left(\gamma_{t}^{*} f_{t}\right) \gamma_{t *} v
$$

where $v$ is our vector field and $f_{t}$ is a smooth function on $t, x, y$ and $\varepsilon$. The solvability of this equation with respect to $f_{t}$ is based on the compatibility of families $\sigma_{1}$ and $\sigma_{2}$ with the family $v$, which immediately implies the compatibility of the families $v$ and $\gamma_{t}$ for all $t \in[0,1]$ due to the definition of $\gamma_{t}$.

The deformation velocity (the index $t$ is omitted) $V$ on the surface $y=0$ (or $\eta=0$ ) has zero at least of the second order. Hence it could be presented in the form

$$
V=\eta^{2}\left(\begin{array}{l}
h(\zeta, \eta, \varepsilon) \\
r(\zeta, \eta, \varepsilon)
\end{array}\right)
$$

with some smooth functions $h$ and $r$. In addition, due to Lemma 2.9 a deformation velocity has to satisfy the equality $\gamma_{*} V=-V$. Substituting that to the form (2.10) we get

$$
\left(\begin{array}{c}
\eta^{2} h(\zeta,-\eta, \varepsilon) \\
-\eta^{2} r(\zeta,-\eta, \varepsilon)
\end{array}\right)=-\left(\begin{array}{c}
\eta^{2} h(\zeta, \eta, \varepsilon) \\
\eta^{2} r(\zeta, \eta, \varepsilon)
\end{array}\right) .
$$

Hence $h(\zeta,-\eta, \varepsilon)=-h(\zeta, \eta, \varepsilon)$ and $r(\zeta,-\eta, \varepsilon)=r(\zeta, \eta, \varepsilon)$, and the functions $h$ and $r$ are odd and even, respectively. Thus locally near the origin they could be presented in the form

$$
h(\zeta, \eta, \varepsilon)=\eta p\left(\zeta, \eta^{2}, \varepsilon\right) \quad \text { and } \quad r(\zeta, \eta, \varepsilon)=q\left(\zeta, \eta^{2}, \varepsilon\right)
$$

with some smooth functions $p$ and $q$.

Thus the velocity $V$ has the form

$$
V(\zeta, \eta, \varepsilon)=\eta^{3} p\left(\zeta, \eta^{2}, \varepsilon\right) \frac{\partial}{\partial \zeta}+\eta^{2} q\left(\zeta, \eta^{2}, \varepsilon\right) \frac{\partial}{\partial \eta} .
$$

Further on the surface $\eta=0$ we have $\gamma_{*} v=-v$ due to the theorem assumptions on compatibility and transversality. Hence near the origin the field $v$ could be written in the form

$$
v(\zeta, \eta, \varepsilon)=\eta l(\zeta, \eta, \varepsilon) \frac{\partial}{\partial \zeta}+m(\zeta, \eta, \varepsilon) \frac{\partial}{\partial \eta},
$$


with some smooth functions $l$ and $m$. The present function $f$ is the sum of its even and odd parts with respect to variable $\eta$, i.e.,

$$
f(\zeta, \eta, \varepsilon)=u\left(\zeta, \eta^{2}, \varepsilon\right)+\eta w\left(\zeta, \eta^{2}, \varepsilon\right),
$$

where $u$ and $w$ are smooth functions. Substituting this expression for $f$ and expressions (2.11) and (2.12) into the terms of the right-hand side of (2.9) we obtain

$$
\begin{aligned}
& \left(f_{t} v\right)(\zeta, \eta, \varepsilon)=\left[u\left(\zeta, \eta^{2}, \varepsilon\right)+\eta w\left(\zeta, \eta^{2}, \varepsilon\right)\right]\left[\eta l(\zeta, \eta, \varepsilon) \frac{\partial}{\partial \zeta}+m(\zeta, \eta, \varepsilon) \frac{\partial}{\partial \eta}\right], \\
& \left(\gamma_{t}^{*} f_{t}\right)(\zeta, \eta, \varepsilon)=f(\zeta,-\eta, \varepsilon)=u\left(\zeta, \eta^{2}, \varepsilon\right)-\eta w\left(\zeta, \eta^{2}, \varepsilon\right), \\
& \left(\gamma_{t *} v\right)(\zeta, \eta, \varepsilon)=-\left(\eta l(\zeta,-\eta, \varepsilon) \frac{\partial}{\partial \zeta}+m(\zeta,-\eta, \varepsilon) \frac{\partial}{\partial \eta}\right), \\
& \left(\left(\gamma_{t}^{*} f_{t}\right) \gamma_{t *} v\right)(\zeta, \eta, \varepsilon)=-\left[u\left(\zeta, \eta^{2}, \varepsilon\right)-\eta w\left(\zeta, \eta^{2}, \varepsilon\right)\right]\left(\eta l(\zeta,-\eta, \varepsilon) \frac{\partial}{\partial \zeta}+m(\zeta,-\eta, \varepsilon) \frac{\partial}{\partial \eta}\right) .
\end{aligned}
$$

Substituting now the expressions (2.11), (2.13) and (2.14) (for $V, f_{t} v$ and $\left(\gamma_{t}^{*} f_{t}\right) \gamma_{t *} v$, respectively) into $(2.9)$ we get

$$
\begin{aligned}
\eta^{3} p\left(\zeta, \eta^{2}, \varepsilon\right) \frac{\partial}{\partial \zeta}+\eta^{2} q\left(\zeta, \eta^{2}, \varepsilon\right) \frac{\partial}{\partial \eta} \\
=\left[u\left(\zeta, \eta^{2}, \varepsilon\right)+\eta w\left(\zeta, \eta^{2}, \varepsilon\right)\right]\left[\eta l(\zeta, \eta, \varepsilon) \frac{\partial}{\partial \zeta}+m(\zeta, \eta, \varepsilon) \frac{\partial}{\partial \eta}\right] \\
\quad+\left[u\left(\zeta, \eta^{2}, \varepsilon\right)-\eta w\left(\zeta, \eta^{2}, \varepsilon\right)\right]\left(\eta l(\zeta,-\eta, \varepsilon) \frac{\partial}{\partial \zeta}+m(\zeta,-\eta, \varepsilon) \frac{\partial}{\partial \eta}\right) \\
=\left[u \eta(l(\zeta, \eta, \varepsilon)+l(\zeta,-\eta, \varepsilon))+w \eta^{2}(l(\zeta, \eta, \varepsilon)-l(\zeta,-\eta, \varepsilon))\right] \frac{\partial}{\partial \zeta} \\
\quad+[u(m(\zeta, \eta, \varepsilon)+m(\zeta,-\eta, \varepsilon))+w \eta(m(\zeta, \eta, \varepsilon)-m(\zeta,-\eta, \varepsilon))] \frac{\partial}{\partial \eta} .
\end{aligned}
$$

Equating the field components from the left and right parts of the last expression we arrive at the following system of linear equations on $u$ and $w$ :

$$
\left\{\begin{array}{l}
u \eta(l(\zeta, \eta, \varepsilon)+l(\zeta,-\eta, \varepsilon))+w \eta^{2}(l(\zeta, \eta, \varepsilon)-l(\zeta,-\eta, \varepsilon))=\eta^{3} p\left(\zeta, \eta^{2}, \varepsilon\right), \\
u(m(\zeta, \eta, \varepsilon)+m(\zeta,-\eta, \varepsilon))+w \eta(m(\zeta, \eta, \varepsilon)-m(\zeta,-\eta, \varepsilon))=\eta^{2} q\left(\zeta, \eta^{2}, \varepsilon\right) .
\end{array}\right.
$$

Dividing here the first equation by $\eta$ we reduce the system to the form

$$
\left\{\begin{array}{l}
u(l(\zeta, \eta, \varepsilon)+l(\zeta,-\eta, \varepsilon))+w \eta(l(\zeta, \eta, \varepsilon)-l(\zeta,-\eta, \varepsilon))=\eta^{2} p\left(\zeta, \eta^{2}, \varepsilon\right), \\
u(m(\zeta, \eta, \varepsilon)+m(\zeta,-\eta, \varepsilon))+w \eta(m(\zeta, \eta, \varepsilon)-m(\zeta,-\eta, \varepsilon))=\eta^{2} q\left(\zeta, \eta^{2}, \varepsilon\right)
\end{array}\right.
$$

The determinant of the matrix of this system is

$$
\left|\begin{array}{cc}
l(\zeta, \eta, \varepsilon)+l(\zeta,-\eta, \varepsilon) & \eta(l(\zeta, \eta, \varepsilon)-l(\zeta,-\eta, \varepsilon)) \\
m(\zeta, \eta, \varepsilon)+m(\zeta,-\eta, \varepsilon) & \eta(m(\zeta, \eta, \varepsilon)-m(\zeta,-\eta, \varepsilon))
\end{array}\right|=\eta^{2}[L+Q(\zeta, \eta, \varepsilon)],
$$

where $L=2\left[l(0,0,0) m_{\eta}(0,0,0)-l_{\eta}(0,0,0) m(0,0,0)\right]$ and $Q$ is a smooth function vanishing at the origin.

Hence, (2.15) is smoothly resolvable with respect to $u$ and $w$ near the origin if $L \neq 0$ because the right-hand side of the system is divided by $\eta^{2}$. But $L$ is not zero due to the compatibility condition of families of involutions and fields. Indeed due to compatibility the area of the parallelogram defined by values of fields $v$ and $\gamma_{*} v$ has the second order zero on the surface $\eta=0$ of fixed points of involutions. Hence the function

$$
\left|\begin{array}{c}
v \\
\gamma_{*} v
\end{array}\right|(\zeta, \eta, \varepsilon)=\left|\begin{array}{cc}
\eta l(\zeta, \eta, \varepsilon) & m(\zeta, \eta, \varepsilon) \\
-\eta l(\zeta,-\eta, \varepsilon) & -m(\zeta,-\eta, \varepsilon)
\end{array}\right|
$$




$$
\begin{aligned}
\text { Davydov A } & \text { Sci China Math November } 2018 \text { Vol. } 61 \text { No.11 } \\
& =-\eta[l(\zeta, \eta, \varepsilon) m(\zeta,-\eta, \varepsilon)-l(\zeta,-\eta, \varepsilon) m(\zeta, \eta, \varepsilon)] \\
& =2 \eta^{2}[L+H(\zeta, \eta, \varepsilon)]
\end{aligned}
$$

where $H$ is a smooth function vanishing at the origin, has the second order zero on this surface. Consequently, $L \neq 0$, and locally near the origin system (2.15) is smoothly resolvable with respect to $u$ and $w$.

Thus Equation (2.9) is smoothly resolvable and germs of families of involutions $\sigma_{1}$ and $\sigma_{2}$ at the origin are strongly $C_{v}^{\infty}$-equivalent.

The reduction theorem is proved.

\section{Classifications of main symbols of mixed type PDEs}

Here, we present the list of known normal forms for typical mixed type partial differential equations on the plane and for families of such equations. We assume that the discriminant of this equation has the non-degenerate zero level, i.e., $d D \neq 0$ when $D=0, D:=b^{2}-4 a c$.

\subsection{History remarks and topological normal forms}

On the type change line for a typical mixed type equation the characteristic direction could rotate by the motion along the line and in a generic case could have tangency with the line. For the Cibrario-Tricomi equation we have not such a tangency, and for the equation $u_{x x}+\left(y+x^{2}\right) u_{y y}=0$ such a tangency takes place at the origin. The Cibrario-Tricomi normal form serves exactly near a point of the type change line, where there is no such tangency.

The typical behaviour of the characteristic net near a point with such a tangency was described in the paper by Pkhakadze and Shestakov [26] (the misprints admitted in this paper were proved in [29]). They analyzed the general first order implicit differential equation $F\left(x, y, y^{\prime}\right)=0$ near a singular point of the lifted direction field. In a generic case such a point is a singular one also for the equation folding, and it is of type Whitney fold. In the paper the author introduced the equation of the first approximation, which corresponds to the Taylor expansion up to order two of the initial equation at the point under consideration. It was proved that for typical coefficient values of the equation of the first approximation the respective point of the lifted direction field is singular of type hyperbolic saddle, node or focus. Also it was shown that these three classical cases of singular points after the equation folding provide three typical singularities of the characteristic net of mixed type equations near the point of tangency of the characteristic direction with the type change line. Now these singularities are called folded saddle, node and focus (see Figure 3).

The normal forms for these three cases were used in 1971 as a model equation by physicists Pilija and Fedorov [25] under the analysis of singularities of the electromagnetic wave field in cold anisotropic plasma with two-dimensional non-homogeneity, while the deduction of these forms was done essentially later on.

In the 70th of the last century there appeared studies by Thom [31], Dara [8], Brodskii (see [1]) and Takens [30], which provided an additional motivation for the analysis of implicit first order differential equations to get their local normal forms for typical cases at least. Dara and Brodskii also rediscovered the Cibrario-Tricomi normal form, and Dara formulated few hypotheses about normal forms for other cases, which later on either have been proved in stronger forms or disproved.

The studies of Thom [31] and Takens [30] were related to geometry and constrained differential equations, and were not directly related to the applications to the analysis of the transonic gas flow, where the Cibrario-Tricomi normal form has already played the important role. But in the connection with the application to the transonic gas flow the topological normal forms of hyperbolic folded saddles, nodes and foci were obtained by Kuzmin [23,24]. The folded singular point is called hyperbolic if the respective singular point of the equation direction field is hyperbolic, i.e., the field could be defined near such a point by the smooth vector field, linearization of which at the point has eigenvalues with nonzero real parts. 


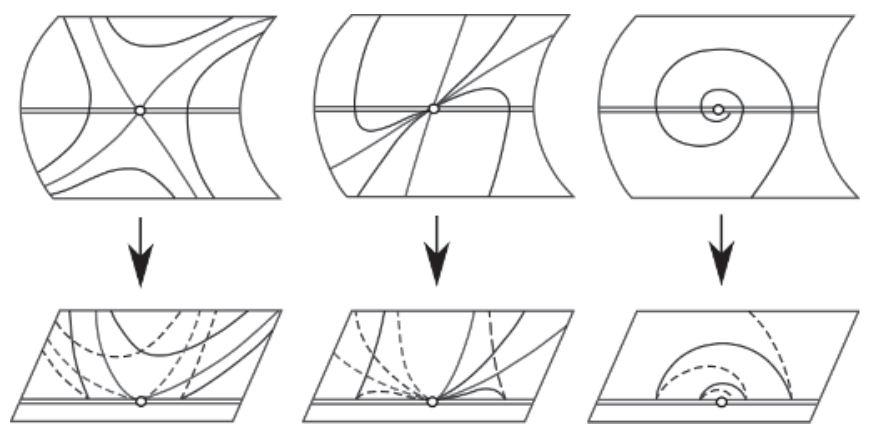

Figure 3 The folded saddle, node and focus, respectively

Theorem 3.1. $\quad$ For a generic smooth equation (1.1) each folded singular point $P$ is hyperbolic, and the characteristic net of this equation is topologically equivalent to the equation

$$
u_{x x}+\left(-y+K x^{2}\right) u_{y y}=0
$$

near the origin with $K=-1,1 / 20$ and 1 for the saddle, node and focus, respectively.

Topological equivalence means up to homeomorphism.

Remark 3.2. Genericity here means "physical", i.e., some property of objects is generic if it takes place for any object from the open everywhere dense subset in the space of objects in an appropriate topology. In our case we consider genericity in the space of triplets $(a, b$ and $c$ in the fine smooth Whitney topology). To verify genericity mentioned in the formulation of Theorem 3.1 it is sufficient to do the following. At the first one needs to check up that this point is non-degenerate for the discriminant, i.e., $D(P)=0, d D(P) \neq 0$. This condition guarantees that the equation folding at the respective point $\tilde{P}$ on the equation surface has singularity of type Whitney fold. At the second one needs to verify that the lifted direction field could be defined near the point $\tilde{P}$ by the smooth vector field with the hyperbolic singular point at $\tilde{P}$ of type saddle, focus or node with exponent $\alpha>1$ (the notion of the exponent is given in the next subsection).

Remark 3.3. The normal form (4.2) also works for smooth families of the equation with the finite dimensional parameter. Namely, if for some value of the parameter we have that the conditions in the previous remark are satisfied then the normal form for a family near the considered folded singular point is exactly form (4.2) up to homeomorphism of the plane, which now continuously depends on the parameter.

As we note above the equation of (3.1) has been already used in [25] to analyze singularities of electromagnetic wave fields. But it is clear that homeomorphism does not guarantee the preservation of main properties of the partial differential equation solutions, and confirmation of such a preservation needs an additional ground. So the topological normal forms for folded singular points could not be directly applied to the analysis of solutions of mixed type PDEs.

\subsection{Smooth normal forms for folded singular points}

The exponent of a hyperbolic singular point of a vector field on the plane is defined to be the ratio of the eigenvalue of the largest modulus of its linearization at the point to the smallest for the saddle and node, and as the modulus of the ratio of the imaginary part of the eigenvalue to the real part for the focus. For a direction field the exponent of a hyperbolic singular point is defined as the one of the respective vector field with the hyperbolic singular point. The exponent is preserved by diffeomorphisms.

Example 3.4. On the plane of variables $u$ and $v$ the germ of a generic $C^{k}$-linearizable with $k \geqslant 1$ direction fields near its non-resonance singular point with exponent $\alpha$ of type saddle, node or focus is $C^{k}$-orbitally equivalent to a germ at the origin of the direction field defined by the vector field

$$
\left(\begin{array}{rr}
0 & 2 \\
-k & 1
\end{array}\right)\left(\begin{array}{c}
u \\
w
\end{array}\right)
$$


with $k=\frac{\alpha}{2(\alpha+1)^{2}}$ for the saddle and node, and with $k=\frac{\alpha^{2}+1}{8}$ for the focus. This vector and involution $(u, w) \mapsto(u,-w)$ are compatible, as it is easy to see.

This compatibility and reduction theorem imply the following theorem.

Theorem 3.5 (See $[2,9,11])$. The germ of the characteristics net of (1.1) at a hyperbolic $C^{\infty}$-linearizable folded singular point with exponent $\alpha$ is the germ at the origin of the equation

$$
u_{x x}+\left(-y+\frac{k x^{2}}{2}\right) u_{x x}=0
$$

in an appropriate smooth coordinate system with the origin at this point.

Here, $k$ is the same as in Example 3.4. The following statement presents the respective normal forms for the characteristic net itself.

Theorem 3.6 (See $[9,11]$ ). The germ of the family of characteristics of $(1.1)$ at a hyperbolic $C^{\infty}$ linearizable folded singular point with exponent $\alpha$ is $C^{\infty}$-diffeomorphic to the germ at the origin of the family of curves which is either

$$
|x \pm \sqrt{y}|^{-\alpha}(x / \alpha \pm \sqrt{y})=c, \quad c \in \mathbb{R}
$$

or

$$
\left(|x \pm \sqrt{y}|^{-\alpha}(x / \alpha \pm \sqrt{y})=c\right) \cup(x \pm \sqrt{y}=0), \quad c \in \mathbb{R}
$$

or else

$$
\left\{\begin{array}{l} 
\pm \alpha^{-1} \sqrt{y}=R \sin \left(\alpha^{-1} \ln R+c\right), \\
x \pm \sqrt{y}=R \cos \left(\alpha^{-1} \ln R+c\right),
\end{array}\right.
$$

for the saddle, node and focus, respectively, where $R=\sqrt{(x \pm \sqrt{y})^{2}+\alpha^{-2} y}$.

The condition of $C^{\infty}$-linearizability in these theorems is not very restricted. Namely, a non-degenerate focus is always $C^{\infty}$-linearizable, and a node is $C^{\infty}$-linearizable if its exponent is not a natural number. Finally, in accordance with the theorem of Segal a saddle with exponent $\alpha$ is $C^{\infty}$-linearizable if the point $(1, \alpha)$ is a point of type $(M, \nu)$, i.e., $\min \left\{\left|1-m_{1}-m_{2} \alpha\right|,\left|\alpha-m_{1}-m_{2} \alpha\right|\right\} \geqslant M /|m|^{\nu}$ for all integer vectors $m=\left(m_{1}, m_{2}\right)$ with non-negative components and with $m_{1}+m_{2} \geqslant 2$. It is known that the measure of the set of points, which for no $M>0$ are points of type $(M, \nu)$, is equal to zero, if $\nu>1$ (see [1,2]).

Thus we have the $C^{\infty}$-linearizability condition for the open everywhere dense subset of nodes and foci, and only for the everywhere dense subset of saddles. The problem is that the resonances for saddles are everywhere dense and generically a resonance saddle is not $C^{\infty}$-linearizable. Normal forms for a generic folded resonance and folded elementary singular points were obtained in $[17,18]$ and $[15,16]$, respectively. The formulation of the respective results is analogous to the classification theorems above. Here, we present the case of the folded resonance saddle to complete the classification of singularities of the characteristic net for a generic mixed type equation near folded singular points, i.e., for the open everywhere dense subsets of (1.1) in the space of equations in fine smooth or sufficiently smooth topology of Whitney.

Theorem 3.7 (See $[17,18]$ ). $\quad$ The germ of the family of characteristics of $(1.1)$ at the folded saddle with exponent $\alpha=-r / q$, where $r$ and $q$ are natural and $r / q$ is an irreducible fraction, is $C^{\infty}$-diffeomorphic to the germ at the origin of the family of characteristics of the equation

$$
u_{x x}+\left[-y-\frac{k}{2} x^{2} \pm x^{r+q+2}+A x^{2(r+q)+2}\right] u_{y y}=0,
$$

where $k=\frac{\alpha}{2(\alpha+1)^{2}}$ as above and $A$ is a real parameter, if the first resonance monomial could not be removed by smooth change of coordinates.

Remark 3.8. One needs to note that the obtained normal forms are not normal forms of the equation but the ones for the characteristics. The equation corresponding to these normal forms is the same up to 
the smooth functions which are equal to zero in the domain $D>0$. For example, if for a generic equation we reduce the germ of its characteristic net to the one of the equation

$$
u_{x x}+\left(-y+\frac{k}{2} x^{2}\right) u_{y y}=0
$$

near the origin, that means that the main symbol of the respective partial differential equation is reduced to the form

$$
(1+a(x, y)) u_{x x}+b(x, y) u_{x y}+\left(-y+\frac{k}{2} x^{2}\right)(1+c(x, y)) u_{y y}=0
$$

with some smooth functions $a, b$ and $c$, which are zero in the domain $D>0$, by smooth change of coordinates and multiplication on the smooth non-vanishing functions. How to remove these $a, b$ and $c$ is an open problem.

\subsection{Smooth family of equations}

Analogous results are also true for smooth families of the equation with the finite dimensional parameter. In order to obtain the respective normal form for folded singular points of such a family it is sufficient to find such a form for the family of the equation direction field, for which the compatible involution could be easily found. Such a pair leads to the respective normal form of the initial family.

For example, a generic focus is always non-resonance. The germ of a smooth family of vector fields at such singular point is locally reduced by $C^{\infty}$-diffeomorphism fibered over the parameter space to the form (3.2), where $k$ smoothly depends on the parameter. Again the field in this form and the involution $(u, w) \mapsto(u,-w)$ are compatible. Hence due to that and the reduction theorem, which was formulated in the parametric form, we have here again the normal form (3.3), in which $k$ depends now on the parameter.

In the recent decades normal forms were also obtained for some generic families of binary differential equations (see $[4-6,13,14]$ ). However, a reasonably complete smooth classification, even for typical oneparameter families, is not obtained yet.

\section{Problems of non-local normal forms}

The question on non-local normal forms of generic linear second order partial differential equations on the plane has appeared naturally after [19], where the structural stability in classical Andronov-PontryaginPeixoto sense was proved for the generic simplest dynamic inequality on the plane

$$
(\dot{x}-v(x, y))^{2}+(\dot{y}-u(x, y))^{2} \leqslant f(x, y)
$$

with the bounded domain of its definition $(=\{f \geqslant 0\})$. Such an inequality describes the simplest motion of the non-inertial object in the plane with the smooth drift $(v, u)$ and maximum proper velocity $\sqrt{f}$. For example, the dynamic inequality

$$
(\dot{x}-2)^{2}+(\dot{y})^{2} \leqslant 1-x^{2}-y^{2}
$$

has admissible velocities in the unit disk $x^{2}+y^{2} \leqslant 1$, and in this disk the set of its orbits (see Figure 1) is structurally stable, i.e., for a sufficiently $C^{2}$-close dynamic inequality we have the same structure of orbits and its family of orbits is reduced to the initial one by homeomorphism of the plane which is close to the identity.

According to Remark 2.3 we see that Figure 1 also provides a structurally stable net of characteristics for some linear second order partial differential equation of mixed type on the plane. That poses the problem to search non-local normal forms for mixed type equations at least for the simplest configurations of the characteristic net like in Figure 1. It is clear that such a form has to account at least exponents of folded foci that we observe in the picture. But due to the results of [4] one could expect even functional modules for such a form. One of the first steps in this direction was done in [21], where the model equation

$$
u_{r r}+(1-r) u_{\phi \phi}=0
$$


was proposed. This equation has the behaviour of the characteristic net near the unit circle, which is analogous to the one provided by the Cibrario-Tricomi equation near its type change line. But in this paper the normal form for the model equation was grounded only in the hyperbolic domain near the circle. Recently as the extension of this normal form to a small neighbourhood of the circle was proposed and one important reduction step was done to provide the full justification this extension, namely, the problem was reduced to the well-known problem of the extension of the solution for the Cauchy problem for the uniformly elliptic equation through the smooth surface. More exactly in the team with Kasten the following statement was proved (the respective paper is submitted for publication).

Let the linear second order partial differential equation with smooth coefficients coincide with (4.2) near the circle $r=1$ in the domain $r \geqslant 1$. Then the main symbol of this equation has the form

$$
(1+a(r, \phi)) d \phi^{2}+b(r, \phi) d r d \phi+(1-r)(1+c(r, \phi)) d r^{2}=0,
$$

where $a, b$ and $c$ are smooth functions, which have zero Taylor expansion at any point of the circle, i.e., flat on the circle. Besides these functions are $2 \pi$-periodic with respect to $\phi$.

Theorem 4.1. The main symbol of (4.3) is reduced to the one of (4.2) in some neighbourhood of the circle $r=1$ by some coordinates change of the form

$$
\tilde{r}=r+R(r, \phi), \quad \tilde{\phi}=\phi+\Phi(r, \phi)
$$

with some smooth functions $R$ and $\Phi$, which are $2 \pi$-periodic in $\phi$ and flat on the circle $r=1$, if near this circle the uniformly elliptic equation

$$
\left[D(r, \phi)\left(1+u_{r}\right)\right]_{r}+\left[D^{-1}(r, \phi) u_{\phi}\right]_{\phi}=0
$$

with a smooth function $D$, which is defined by $a, b, c$ and is equal to 1 in the domain $r \geqslant 1$, has a smooth solution, which is zero in this domain.

As we have already noted above this theorem reduces the problem of deriving the necessary normal form to the famous problem about the existence of a smooth extension for a solution of the Cauchy problem for uniformly elliptic equations through a hypersurface. For (4.5) such an extension is unique (see [22]). But we have not found in the literature any clear statement about the existence of such an extension for the equation of our type. But due to the domain, in which such an extension is needed and could be taken like a small narrow neighborhood of the circle such an extension has to exist (see [20]).

To prove Theorem 4.1 the following statement is useful.

Lemma 4.2. If a smooth function $f$ on $m+1$ real variables $x \in \mathbb{R}$ and $y \in \mathbb{R}^{m}$ is flat on the plane $x=0$ then for any positive real $\nu$ there exists a smooth function $F$ on $m+1$ real variables such that for $x \geqslant 0$ we have

$$
f(x, y)=F\left(x^{\nu}, y\right) .
$$

Indeed, for $z \neq 0$ define $F(z, y):=f\left(|z|^{1 / \nu}, y\right)$. It is clear that outside the hyperplane $z=0$ the function $F$ is smooth. But $f$ is flat on $x=0$, and hence the function $F$ is also flat on $z=0$. Thus the function $F$ is smooth, and the statement of the lemma is true.

Using this statement, we rewrite (4.3) near the circle $r=1$ in the domain $r \leqslant 1$ in the form

$$
(1+A(z, \phi)) d \phi^{2}+B(z, \phi) d z d \phi+\frac{4}{9}(1+C(z, \phi)) d z^{2}=0,
$$

with $z:=(1-r)^{3 / 2}$ and some smooth functions $A, B$ and $C$, which are flat on $z=0$ and $2 \pi$-periodic in $\phi$. After the division on the coefficient $1+A$ this equation takes the form

$$
d \phi^{2}+B(z, \phi) d z d \phi+\frac{4}{9}(1+C(z, \phi)) d z^{2}=0,
$$

with some new functions $B$ and $C$ with the same properties. To prove the theorem we work with this equation in the domain $z \geqslant 0$ near the type change line and control that the coordinate changes used are identical up to the adding of smooth functions, which are flat on this line. 
To remove the function $B$ take a new coordinate $\tilde{\phi}$, which is near the circle $z=0$ and is the solution of the equation

$$
2 \tilde{\phi}_{z}-B \tilde{\phi}_{\phi}=0
$$

with the initial value $\phi$ on the circle. This solution exists and is unique because the line has no characteristic points of the equation (see [1]). Also this solution equals $\phi$ up to a flat function on the circle because the function $B$ is flat on it.

So from now on we work with (4.7) with $B \equiv 0$ and in the form

$$
d \phi^{2}+(1+C(z, \phi)) d z^{2}=0,
$$

which could be obtained from the form (4.7) with $B \equiv 0$ by rescaling of coordinate $z$.

To prove Theorem 4.1 it is sufficient in the domain $z \geqslant 0$ near the circle to find coordinates of (4.4), i.e.,

$$
\tilde{z}=z+Z(z, \phi), \quad \tilde{\phi}=\phi+\Phi(z, \phi)
$$

with some smooth functions $Z$ and $\Phi$, which are $2 \pi$-periodic with respect to the second argument and flat on the circle $z=0$, such that in the new coordinates Equation (4.8) takes the form ("tilde" is omitted)

$$
d \phi^{2}+d z^{2}=0
$$

up to multiplication on a smooth non-vanishing function. Indeed, the last equation by transformations

$$
\hat{\phi}=\phi, \quad \frac{2}{3}(1-\hat{r})^{3 / 2}=z
$$

is reduced in the domain $z \geqslant 0$ near the circle $z=0$ to the form

$$
d \hat{\phi}^{2}+(1-\hat{r}) d \hat{r}^{2}=0
$$

in the domain $\hat{r} \leqslant 1$ near the circle $\hat{r}=1$. Due to the flatness of functions $\Phi$ and $Z$ on the circle $\tilde{z}=0$ the final change of coordinates

$$
(r, \phi) \mapsto(\hat{r}, \hat{\phi})
$$

is smooth in the domain $r \leqslant 1$ near the circle $r=1$ and could be smoothly extended to some neighborhood of this circle as the identical map in the domain $r \geqslant 1$.

Thus, it is sufficient to prove the existence of coordinates (4.4) with properties requested. To construct such coordinate we calculate from (4.9) the differentials

$$
d z=\frac{\left(1+\Phi_{\phi}\right) d \tilde{z}-Z_{\phi} d \tilde{\phi}}{\Delta}, \quad d \phi=\frac{-\Phi_{z} d \tilde{z}+\left(1+Z_{z}\right) d \tilde{\phi}}{\Delta},
$$

where

$$
\Delta=\left(1+Z_{z}\right)\left(1+\Phi_{\phi}\right)-Z_{\phi} \Phi_{z},
$$

and substitute them to (4.8). Multiplying the obtained equation by $\Delta^{2}$ we get

$$
\begin{aligned}
& {\left[\left(1+Z_{z}\right)^{2}+(1+C) Z_{\phi}^{2}\right] d \tilde{\phi}^{2}-2\left[\left(1+Z_{z}\right) \Phi_{z}+(1+C)\left(1+\Phi_{\phi}\right) Z_{\phi}\right] d z d \phi} \\
& \quad+\left[\Phi_{z}^{2}+(1+C)\left(1+\Phi_{\phi}\right)^{2}\right]=0 .
\end{aligned}
$$

One needs to find smooth $Z$ and $\Phi$ being flat on $z=0$ such that in the last equation the middle coefficient is zero and two others are equal identically. That leads to the system

$$
\left\{\begin{array}{l}
\left(1+Z_{z}\right) \Phi_{z}+(1+C)\left(1+\Phi_{\phi}\right) Z_{\phi}=0, \\
\left(1+Z_{z}\right)^{2}+(1+C) Z_{\phi}^{2}=\Phi_{z}^{2}+(1+C)\left(1+\Phi_{\phi}\right)^{2} .
\end{array}\right.
$$

Substituting from the first equation of this system

$$
\Phi_{z}=-\frac{(1+C)\left(1+\Phi_{\phi}\right) Z_{\phi}}{1+Z_{z}}
$$


to the second we get the following equation:

$$
\left(1+Z_{z}\right)^{2}+(1+C) Z_{\phi}^{2}=\left[\frac{(1+C)\left(1+\Phi_{\phi}\right) Z_{\phi}}{1+Z_{z}}\right]^{2}+(1+C)\left(1+\Phi_{\phi}\right)^{2}
$$

or

$$
\left(1+Z_{z}\right)^{2}+(1+C) Z_{\phi}^{2}=\left[\frac{(1+C)\left(1+\Phi_{\phi}\right)}{1+Z_{z}}\right]^{2}\left[(1+C) Z_{\phi}^{2}+\left(1+Z_{z}\right)^{2}\right] .
$$

We search a function $Z$ which is flat on the circle $z=0$. For such a function the expression on the lefthand side of the last equation is not zero near this circle. Dividing the last equation by this expression and using the (needed) flatness of $Z$ and $\Phi$ we get the equation

$$
\sqrt{1+C}\left(1+\Phi_{\phi}\right)=1+Z_{z}
$$

This equation together with the first equation of (4.10) leads to

$$
\left\{\begin{array}{l}
-\Phi_{z}=\sqrt{1+C} Z_{\phi}, \\
1+\Phi_{\phi}=\frac{1}{\sqrt{1+C}}\left(1+Z_{z}\right) .
\end{array}\right.
$$

The integrability condition for this system gives the following second order partial differential equation:

$$
\left(\sqrt{1+C} Z_{\phi}\right)_{\phi}+\left(\frac{1}{\sqrt{1+C}}\left(1+Z_{z}\right)\right)_{z}=0
$$

where the function $D, D(z, \phi)=\sqrt{1+C(z, \phi)}$, is smooth and equal to 1 in the domain $z \leqslant 0(r \geqslant 1)$. In this domain near this circle the last equation has zero solution $Z=0$. Hence, if this solution could be smoothly extended to a neighbourhood of this circle then the extension is flat on the circle itself and the needed change of coordinates exists.

Thus the statement of Theorem 4.1 is true.

Acknowledgements This work was supported by the Ministry of Education and Science of the Russian Federation (Grant No. 1.638.2016/FPM).

Open access funding provided by International Institute for Applied Systems Analysis (IIASA).

Open Access This article is distributed under the terms of the Creative Commons Attribution 4.0 International License (http://creativecommons.org/licenses/by/4.0/), which permits use, duplication, adaptation, distribution and reproduction in any medium or format, as long as you give appropriate credit to the original author(s) and the source, provide a link to the Creative Commons license and indicate if changes were made.

\section{References}

1 Arnold V I. Geometrical Methods in the Theory of Ordinary Differential Equations. New York: Springer-Verlag, 1983

2 Arnold V I, Ilyashenko Y S. Ordinary differential equations. Encyclopaedia Math Sci, 1988, 1: 1-148

3 Arnold V I, Varchenko A N, Gusein-Sade S M. Singularities of Differentiable Mapping, Volume 1. Monographs in Mathematics, vol. 82. Boston: Birkhäuser, 1985

4 Bogaevsky I A. Implicit ordinary differential equations: Bifurcations and sharpening of equivalence. Izv Math, 2014, 78: $1063-1078$

5 Bruce J W, Tari F. Generic 1-parameter families of binary differential equations of Morse type. Discrete Contin Dyn Syst, 1997, 3: 79-90

6 Bruce J W, Tari F, Fletcher G J. Bifurcations of binary differential equations. Proc Roy Soc Edinburgh Sect A, 2000, 130: 485-506

7 Cibrario M. Sulla reduzione a forma canonica delle equazioni lineari alle derivative parzialy di secondo ordine di tipo misto. Rend Lombardo, 1932, 65: 889-906

8 Dara L. Singularities generiques des equations differentielles multiformes. Bol Soc Bras Mat, 1975, 6: 95-128

9 Davydov A A. The normal form of a differential equation that is not solved with respect to derivative, in the neighbourhood of its singular point. Funct Anal Appl, 1985, 19: 81-89 
10 Davydov A A. Structural stability of control systems on orientable surfaces. Mat Sb, 1992, 72: 1-28

11 Davydov A A. Qualitative Theory of Control Systems. Translations of Mathematical Monographs, vol. 141. Providence: Amer Math Soc, 1994

12 Davydov A A, Diep L T T. Normal forms for families of linear equations of mixed type near non-resonant folded singular points. Russian Math Surveys, 2010, 65: 984-986

13 Davydov A A, Diep L T T. Reduction theorem and normal forms of linear second order mixed type PDE families in the plane. TWMS J Pure Appl Math, 2011, 2: 44-53

14 Davydov A A, Ishikawa G, Izumiya S, et al. Generic singularities of implicit systems of first order differential equations on the plane. Jpn J Math, 2008, 3: 93-119

15 Davydov A A, Ortiz-Bobadilla L. Smooth normal forms of folded elementary singular points. J Dyn Control Syst, 1995, 1: 463-483

16 Davydov A A, Ortiz-Bobadilla L. Normal forms of folded elementary singular points. Russian Math Surveys, 1995, 50: $1260-1261$

17 Davydov A A, Rosales-Gonzales E. Complete classification of generic linear second-order partial differential equations in the plane. Dokl Math, 1996, 350: 151-154

18 Davydov A A, Rosales-Gonzales E. Smooth normal forms of folded resonance saddles and nodes and complete classification of generic linear second order PDE's on the plane. In: International Conference on Differential Equations. Singapore: World Scientific, 1998, 59-68

19 Grishina Y A, Davydov A A. Structural stability of simplest dynamical inequalities. Proc Steklov Inst Math, 2007, 256: $80-91$

20 Hormander L. On the theory of general partial differential operators. Acta Math, 1955, 94: 161-248

21 Kasten J A. Solvability of the boundary value problem for a Tricomi type equation in the exterior of a disk. J Math Sci, 2013, 188: 268-272

22 Kondratiev V A, Landis E M. Qualitative theory of second order linear partial differential equations. Itogi Nauki i Tekhniki Ser Sovrem Probl Mat Fund Napr, 1988, 32: 99-215

23 Kuzmin A G. On the behavior of the characteristics of equations of mixed type near the line of degeneracy. Differ Uravn, 1981, 17: 2052-2063

24 Kuzmin A G. Non-Classical Equations of Mixed Type and Their Applications in Gas Dynamics. International Series of Numerical Mathematics, vol. 109. Basel: Birkhäuser, 1992

25 Pilija A D, Fedorov V I. Singularities of electromagnetic wave field in cold anisotropic plasma with two-dimensional non-homogeneity. J Exp Theor Phys, 1971, 60: 389-400

26 Pkhakadze A V, Shestakov A A. On the classification of the singular points of a first order differential equation not solved for the derivative. Mat Sb, 1959, 49: 3-12

27 Rassias J M. Lecture Notes on Mixed Type Partial Differential Equations. Singapore: World Scientific, 1990

28 Smirnov M M. Equations of Mixed Type. Translations of Mathematical Monographs, vol. 51. Providence: Amer Math Soc, 1978

29 Sokolov P V. On the paper of A. V. Phadadze and A. A. Shestakov "On the classification of the singular points of a first order differential equation not solved for the derivative" (in Russian). Mat Sb, 1961, 53: 541-543

30 Takens F. Constrained equations: A study of implicit differential equations and their discontinuous solutions. In: Structural Stability, the Theory of Catastrophes, and Applications in the Sciences. Berlin-Heidelberg: Springer, 1976, $143-234$

31 Thom R. Sur les equations differentielles multiformes et leurs integrales singulieres. Bol Soc Bras Mat, 1972, 3: 1-11

32 Tricomi F. Sulle equazioni lineari alle derivate partziali di secondo ordine di tipo misto. Rend Reale Accad Lincei, 1923, 14: 134-247 